Running Head: Ownership and Self-Prioritization

\title{
How Prioritized is Self-Prioritization During Stimulus Processing?
}

\author{
Johanna K. Falbén, ${ }^{1}$ Marius Golubickis, ${ }^{2}$ Ruta Balseryte, ${ }^{1}$ \\ Linn M. Persson, ${ }^{1}$ Dimitra Tsamadi, ${ }^{1}$ Siobhan Caughey, ${ }^{1}$ C. Neil Macrae ${ }^{1}$ \\ ${ }^{1}$ School of Psychology, University of Aberdeen, Aberdeen, Scotland, UK \\ ${ }^{2}$ Department of Psychology, University of Toronto, Toronto, Canada
}

Word Count: 3000

Address Correspondence to:

Johanna Falbén

School of Psychology

University of Aberdeen

Aberdeen AB24 3FX

Scotland, UK

Email: johanna.falben.12@aberdeen.ac.uk 


\begin{abstract}
Recent research has suggested that self-relevance automatically enhances stimulus processing (i.e., the self-prioritization effect). Notably, information associated with one's self elicits faster responses than comparable material associated with other targets (e.g., friend, stranger). Challenging the assertion that self-prioritization is an obligatory process, here we hypothesized that self-relevance only facilitates performance when task sets draw attention to previously formed target-object associations. The results of two experiments were consistent with this viewpoint. Compared with arbitrary objects owned by a friend, those owned by the self were classified more rapidly when participants were required to report either the owner or identity of the items (i.e., semantic task set). In contrast, self-relevance failed to facilitate performance when participants judged the orientation of the stimuli (i.e., perceptual task set). These findings demonstrate the conditional automaticity of self-prioritization during stimulus processing.
\end{abstract}

Keywords: self-relevance, self-prioritization, ownership, automaticity, task sets 
How Prioritized is Self-Prioritization During Stimulus Processing?

Commonplace in the psychological literature is the demonstration that self-relevance affords significant benefits during stimulus processing. Compared to items associated with other people, self-relevant material is easier to detect, judge, and remember (Bargh \& Pratto, 1986; Keyes \& Brady, 2010; Kuiper \& Rogers, 1979; Shapiro, Caldwell, \& Sorensen, 1997; Symons \& Johnson, 1997). Overcoming the stimulus confounds (e.g., own face/name familiarity) that undermined previous work on this topic, Sui, He, and Humphreys (2012) showed that the advantages of selfrelevance extend even to inconsequential stimuli that have been linked with the self. After coupling geometric shapes with person labels $($ circle $=$ you, triangle $=$ best friend, square $=$ stranger $)$, participants' perceptual-matching judgments (i.e., do shape-label stimulus pairs match the learned associations?) were fastest and most accurate for stimulus pairs associated with the self (vs. best friend or stranger), a phenomenon they termed the self-prioritization effect (Sui et al., 2012; Sui, Liu, Moverach, \& Humphreys, 2013; Sui, Sun, Peng, \& Humphreys, 2014). The favored explanation for this effect is that the mind is finely tuned to information that pertains to the self, such that — via bottom-up attentional capture — self-relevance automatically facilitates task performance (Humphreys \& Sui, 2016; Sui \& Humphreys, 2017). But is this conclusion warranted?

Inspection of the available evidence suggests that the benefits of self-relevance may be far from obligatory. In part, difficulties derive from an over-reliance on perceptual-matching tasks to investigate the effects of self-relevance on stimulus processing (Reuther \& Chakravarthi, 2017). A problematic feature of this methodology is that, on a trial-by-trial basis, participants must explicitly monitor the self-relevance (or otherwise) of the stimuli — indeed perceptual matching cannot be performed successfully otherwise. Crucially, when this task requirement is removed, selfprioritization no longer emerges (Siebold, Weaver, Donk, \& van Zoest, 2016; Stein, Siebold, \& Zoest, 2016; Wade \& Vickery, in press). For example, using breaking continuous flash suppression 
(b-CFS) to investigate the ease with which stimuli access visual awareness, Stein et al. (2016) reported no effect of self-association on the time taken for objects (i.e., Gabors) to overcome interocular suppression (cf. Macrae, Visokomogilski, Cunningham, \& Sahraie, 2017). Interestingly, however, in a prior perceptual-matching task (i.e., Gabor-label pairings), a standard selfprioritization effect was observed. Thus, beyond explicit shape-label matching tasks, these findings challenge the assertion that self-prioritization is an unconditionally automatic process.

One possibility is that self-prioritization is modulated by the task set that is adopted during stimulus processing (De Houwer, Hermans, Rothermund, \& Wentura, 2002; Henik, Friedrich, \& Kellog, 1983; Klauer \& Musch, 2002; Maxfield, 1997). Revised theories of automaticity contend that automatic processes only occur when the cognitive system is configured in particular ways (Kiefer, 2007; Moors \& De Houwer, 2006). In other words, automatic processes are conditional on specific triggering conditions (Bargh, 1989). Self-prioritization likely operates in this way. Specifically, while self-relevance facilitates performance when task sets yield access to targetobject associations in memory (Macrae et al., 2017; Sui et al., 2012), when, in contrast, they demand only the perceptual appraisal of an item (i.e., target-object associations are task irrelevant) self-prioritization does not emerge (Siebold et al., 2016; Stein et al., 2016). Operating in this way, self-prioritization is consistent with Hommel's (2004) Theory of Event Coding, whereby targetobject associations in memory are differentially activated as a function of task set. Whereas explicit assessments of stimulus relevance would yield access to the complete event file, hence trigger selfprioritization; perceptual appraisal of a stimulus would fail to activate any part of the event file, thus prevent self-prioritization from arising.

Endorsing the conditional automaticity of mental life, here we examined the hypothesis that self-prioritization is contingent on the task set that is operating during stimulus processing (i.e., selfprioritization is under attentional control). In two experiments, using an object-ownership paradigm (Golubickis, Falbén, Cunningham, \& Macrae, 2018), participants were presented with items (e.g., 
pencils or pens) that ostensibly belonged either to the self or a best friend. Their task was simply to classify the objects (i.e., owned-by-self vs. owned-by-friend) as quickly and accurately as possible. Participants completed three blocks of trials, with each block requiring a different judgment to be undertaken on the stimuli. Replicating previous research (Golubickis et al., 2018), one block probed the ownership of the items, a task set (i.e., overt judgment of self-relevance) that has been shown to produce a self-prioritization effect (i.e., self-owned objects are classified more rapidly than friendowned objects). In this condition, both components of the event file (i.e., target-object association) would be activated (Hommel, 2004). For comparison, two additional blocks of trials required participants to report either the identity (i.e., pencil or pen - semantic task set) or orientation (i.e., vertical or horizontal - perceptual task set) of the objects. By activating one element of the event file (i.e., object identity), a semantic task set was also expected to trigger self-prioritization. ${ }^{1}$ In contrast, self-prioritization was not expected to emerge when attention was directed to a perceptual analysis of the objects, as target-object associations are task relevant.

\section{Experiment 1}

\section{Method}

\section{Participants and Design}

Forty undergraduates $\left(13\right.$ males, $\left.M_{\text {age }}=20.61, S D=1.96\right)$ took part in the research ${ }^{2}$ All participants had normal or corrected-to-normal visual acuity. Informed consent was obtained from participants prior to the commencement of the experiment and the protocol was reviewed and approved by the Ethics Committee at the School of Psychology, University of Aberdeen. The

\footnotetext{
${ }^{1}$ Processing the identity of the objects would be sufficient to activate the complete event file (i.e., target-object association; Hommel, 2004; Frings, Rothermund, \& Wentura, 2007), hence trigger self-prioritization.

${ }^{2}$ Based on related research (Golubickis et al., 2018), G*Power revealed a requirement of 36 participants for each experiment. An additional $\sim 10 \%$ were recruited to allow for drop out.
} 
experiment had a 3 (Judgment: ownership vs. identity vs. orientation) X 2 (Owner: self vs. friend) repeated measures design.

\section{Stimulus Materials and Procedure}

Participants arrived at the laboratory individually, were greeted by an experimenter, seated in front of a desktop computer, and informed that the experiment comprised an objectcategorization task featuring two categories of objects, pencils and pens (Golubickis et al., 2018). Next participants were told that, prior to the commencement of the task, the computer would randomly assign one category of object to them (i.e., owned-by-self) and the other category of object to their best friend (i.e., owned-by-friend). That is, participants would own all the items (i.e., pencils or pens) from one of the categories, and their best friend would own all the items from the other category. They then pressed the spacebar on the keyboard and text appeared revealing who had been assigned the pencils and pens, respectively (i.e., you $=$ pens, friend $=$ pencils). Assignment of the objects to self and friend was counterbalanced across the sample.

The experiment comprised three blocks of trials, with a different stimulus-related judgment required in each block. In one block, participants reported whether the object presented on the screen (i.e., pencil or pen) belonged to them or their friend (i.e., ownership judgment). In another block, whether the object was a pencil or a pen (i.e., identity judgment) and, in a final block, whether the object was displayed vertically or horizontally on the screen (i.e., orientation judgment). The order of the judgment tasks was counterbalanced across the sample. Before the start of each block, the experimenter explained that participants would be presented with a series of pictures of individual pencils and pens and their task was simply to categorize each object (via a button press), as quickly and accurately as possible, according to the specific judgment task. Responses were given using two buttons on the keyboard (i.e., N \& M). Key-response mappings were counterbalanced across participants and across the judgment tasks, and the response labels 
'mine' and 'friend,' 'pencil' and 'pen,' and 'vertical' and 'horizontal' were located above the relevant response buttons during each block.

Each trial began with the presentation of a central fixation cross for $500 \mathrm{~ms}$, followed by the picture of a pencil or pen for $100 \mathrm{~ms}$. After each object was presented, the screen turned blank until participants responded. Following each response, the fixation cross reappeared and the next trial commenced. The two categories of stimuli comprised photographs of 28 unique objects (14 pencils and 14 pens) that were taken from Google images and edited using Photoshop CS6, such that each pencil or pen was presented in both horizontal and vertical orientations throughout the experiment. Images were 214 x 214 pixels in size, greyscale, and matched for luminance. Participants initially performed 8 practice trials, followed by a block of 196 experimental trials in which all stimuli occurred equally often (i.e., seven times per block) in a random order. In total, there were 588 trials, with 196 trials in each block (i.e., judgment task), and 98 trials in each condition (i.e., mine vs. friend, pencil vs. pen, vertical vs. horizontal). On completion of the task, participants were debriefed, thanked, and dismissed.

\section{Results and Discussion}

Responses faster than $200 \mathrm{~ms}$ were excluded from the analysis, eliminating less than $1 \%$ of the overall number of trials. Table 1 shows the accuracy and response time (RT) data. A 2-level multilevel model was used to examine the RT and accuracy data. Analyses were conducted with the R package 'Imer4' (Pinheiro, Bates, DebRoy, Sarkar, \& R Core Team, 2015). In a series of mixedeffects linear regression models, the main effects of Judgment (ownership vs. identity vs. orientation) and Owner (self vs. friend), and the Judgment X Owner interaction were examined. The analysis yielded main effects of Judgment $\left(b=-.050, S E=.001, t=-47.25, p<.001, \mathrm{R}_{\beta}^{2}=.2163\right)$ and Owner $\left(b=-.004, S E=.001, t=-4.66, p<.001, \mathrm{R}_{\beta}^{2}=.0021\right)$, and a significant Judgment $\mathrm{X}$ Owner interaction $\left(b=-.003, S E=.001, t=-2.73, p=.006, \mathrm{R}_{\beta}^{2}=.0007\right)$ on the speed of 
participants' responses. Further analysis of the interaction revealed that RTs were faster for selfowned than friend-owned objects on judgments of ownership $(b=-.007, S E=.001, t=-4.73, p$ $\left.<.001, \mathrm{R}_{\beta}^{2}=.0066\right)$ and identity $\left(b=-.004, S E=.001, t=-2.46, p=.014, \mathrm{R}_{\beta}^{2}=.0018\right)$. No such effect emerged on judgments of stimulus orientation $\left(b=-.001, S E=.001, t=-0.83, p=.404, \mathrm{R}_{\beta}^{2}\right.$ $=.0001)$.

A 2-level multilevel logistic regression model on the accuracy of participants' responses yielded a main effect of Judgment $\left(b=-.268, S E=.027, z=-9.87, p<.001, \mathrm{R}_{\beta}^{2}=.0085\right)$ and a significant Judgment X Owner interaction $\left(b=-.057, S E=.027, z=-2.08, p=.037, \mathrm{R}_{\beta}^{2}=.0004\right)$. Further analysis of the interaction revealed that, on ownership judgments, accuracy was greater for friend-owned than self-owned items $\left(b=-.086, S E=.036, z=-2.38, p=.017, \mathrm{R}_{\beta}^{2}=.0015\right)$. No such effect emerged on judgments of identity $\left(b=-.040, S E=.039, z=-1.03, p=.305, \mathrm{R}_{\beta}^{2}=.0001\right)$ or orientation $\left(b=.043, S E=.047, z=.93, p=.353, \mathrm{R}_{\beta}^{2}=.0002\right)$.

These results confirm that self-prioritization is dependent on the task set that is operating during stimulus processing. Specifically, when judgments pertained to the ownership or identity of objects a self-prioritization effect was observed, such that responses were faster to items associated with the self than items associated with a friend. In contrast, adoption of perceptual task set (i.e., stimulus orientation) eliminated this effect. In our second experiment, using different objects, we sought to replicate these findings. 
Table 1. Mean reaction times (RT) and accuracy as a function of judgment type and owner (Expts. 1 \& 2).

Judgment $\quad$ Owner $\quad$ Mean RT (ms) Accuracy (\%)

$\begin{array}{llll}\text { Experiment } 1 & & & \\ \text { ownership } & \text { self } & 516(144) & 88(33) \\ & \text { friend } & 529(149) & 90(31) \\ \text { identity } & \text { self } & 510(147) & 87(34) \\ & \text { friend } & 515(147) & 87(33) \\ \text { orientation } & \text { self } & 421(135) & 94(24) \\ & \text { friend } & 423(139) & 93(25)\end{array}$

Experiment 2

\begin{tabular}{llll} 
ownership & self & $431(133)$ & $88(33)$ \\
\multirow{2}{*}{ identity } & friend & $440(133)$ & $90(30)$ \\
& self & $428(126)$ & $87(34)$ \\
\multirow{2}{*}{ orientation } & friend & $435(128)$ & $87(33)$ \\
& self & $399(129)$ & $93(24)$ \\
& friend & $394(123)$ & $93(24)$
\end{tabular}

Note. Standard deviations appear within parentheses.

\section{Experiment 2}

\section{Method}

\section{Participants and Design}

Forty undergraduates ( 9 males, $\left.M_{\text {age }}=22.55, S D=7.23\right)$ took part in the research. All participants had normal or corrected-to-normal visual acuity. Three participants (1 male) failed to follow the instructions by responding with invalid key presses, thus were excluded from the statistical analysis. Informed consent was obtained from participants prior to the commencement of 
the experiment and the protocol was reviewed and approved by the Ethics Committee at the School of Psychology, University of Aberdeen. The experiment had a 3 (Judgment: ownership vs. identity vs. orientation) X 2 (Owner: self vs. friend) repeated measures design.

\section{Stimulus Materials and Procedure}

The study closely followed Experiment 1, but with an important modification. On this occasion, the to-be-judged stimuli were forks and spoons. On completion of the task, participants were debriefed, thanked, and dismissed.

\section{Results and Discussion}

Responses faster than $200 \mathrm{~ms}$ were excluded from the analysis, eliminating less than $1 \%$ of the overall number of trials. Table 1 shows the accuracy and RT data. A 2-level multilevel model analysis on RTs yielded main effects of Judgment $\left(b=.019, S E=.001, t=19.02, p<.001, \mathrm{R}_{\beta}^{2}\right.$ $=.0369)$ and Owner $\left(b=-.002, S E=.001, t=-2.28, p=.023, \mathrm{R}_{\beta}^{2}=.0005\right)$, and a significant Judgment X Owner interaction $\left(b=-.003, S E=.001, t=-3.35, p<.001, \mathrm{R}_{\beta}^{2}=.0012\right)$. Further analysis of the interaction revealed that RTs were significantly faster for self-owned than friendowned objects on judgments of ownership $\left(b=-.005, S E=.001, t=-3.27, p=.001, \mathrm{R}_{\beta}^{2}=.0033\right)$ and identity $\left(b=-.003, S E=.001, t=-2.20, p=.028, \mathrm{R}_{\beta}^{2}=.0015\right)$. No such effect emerged on judgments of stimulus orientation $\left(b=.002, S E=.001, t=1.60, p=.110, \mathrm{R}_{\beta}^{2}=.0008\right)$. A 2-level multilevel logistic regression model on the accuracy of participants' responses yielded no significant effects.

These results directly replicate Experiment 1 . Whereas judgments of ownership and identity elicited a self-prioritization effect during object processing, reporting the orientation of an object 
was insensitive to the self-relevance of the item. This provides further evidence that selfprioritization does not occur unconditionally, but instead requires controlled access to target-object associations. It should be noted, however, that the absence of a self-prioritization effect when judging the orientation of a stimulus may potentially be attributable to a different factor, a floor effect in RTs (i.e., responses were fastest in this condition). To address this issue, we used a bootstrapping procedure to examine the distribution characteristic of judgments in each condition in Experiment 2 (i.e., self \& friend), combining accuracy and RT performance (Davison \& Hinkley, 1997). For each participant, in each condition, accuracy and RT were paired as a single data point $(x, y)$. A bootstrapped data set was then created by re-sampling the data with replacement, keeping the sample size as the number of participants. The mean of this bootstrapped data set was then calculated and plotted as a single data point in the distribution $(x, y)$. This procedure was repeated 20,000 times to estimate the population mean and variation for each condition. As can be seen in Figure 1, the bootstrapped sample mean observations for each judgment overlap almost completely, thereby indicating that the failure to observe a self-prioritization effect in the perceptual condition was not because of a floor effect in response times (i.e., a self-prioritization effect was observed in the other two overlapping conditions). 

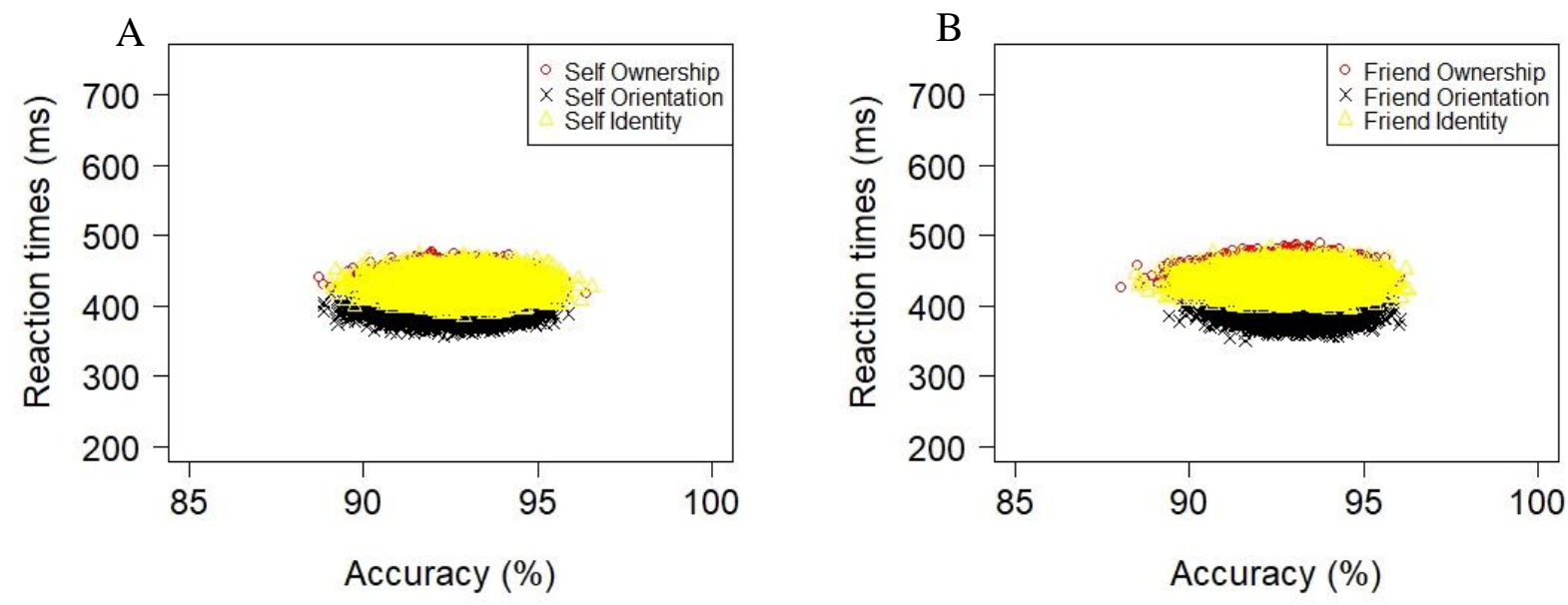

Figure 1. Distribution of bootstrapped sample means for self (A) and friend (B). The $x$-axis represents the accuracy rates and the $y$-axis the reaction times.

\section{General Discussion}

Despite abundant evidence for the prioritization of self-relevant stimuli during decisional processing (Sui \& Humphreys, 2017), questions remain regarding the cognitive status of this effect. In an attempt to elucidate this issue, here we demonstrated the conditional automaticity of selfprioritization. At least in the context of object ownership, self-relevance only facilitated performance when task sets (e.g., ownership or identity) directed attention (i.e., yielded access) to previously formed target-object associations (Hommel, 2004). When emphasis switched instead to a perceptual appraisal of the stimuli, self-prioritization was not observed (Siebold et al., 2016; Stein et al., 2016). At a minimum, therefore, self-prioritization appears to demand the semantic appraisal of stimuli to access target-object relations in memory (Kiefer, 2007; Maxfield, 1997).

The current findings sit comfortably with theoretical accounts of automaticity which assert that automatic processes are fundamentally dependent on top-down factors that regulate cognitive functioning (Kiefer, 2007; Moors \& De Houwer, 2006; Naccache et al., 2002). Here we showed that self-prioritization was contingent on the operation of task sets that directed attention to previously 
formed target-object relations (Hommel, 2004). Allotting attention to the perceptual characteristics of an object, in contrast, was not sufficient to access this information and facilitate performance. That self-prioritization is under attentional control makes functional sense. Although one's possessions clearly occupy a position of prominence in everyday life, absent specific processing objectives (e.g., locating one's car in the parking lot, selecting shoes to wear on a date) these items frequently have no immediate applicability. It would therefore be somewhat cumbersome if the mind were to unconditionally prioritize currently inconsequential objects based only on their selfrelevance. Under attentional control, self-prioritization can facilitate thinking and doing in a flexible, strategic manner.

In sum, the current research demonstrated the conditional automaticity of self-prioritization. A number of important questions remain, however. In particular, would the results reported here emerge in non-ownership contexts and with different stimulus materials? Also, it is unlikely that attentional sensitization to target-object associations rely on the instructed application of specific task sets; similar effects likely emerge via non-conscious pathways (Kiefer, Adams, \& Zovko, 2012; Moors \& De Houwer, 2006). Extending the current investigation, further research will be required to clarify exactly when self-prioritization facilitates stimulus processing. 


\section{References}

Bargh, J. A. (1989). Conditional automaticity: Varieties of automatic influence in social perception and cognition. In J. S. Uleman \& J. A. Bargh (Eds.), Unintended Thought (pp. 3-51). New York, NY: Guilford Press.

Bargh, J. A., \& Pratto, F. (1986). Individual construct accessibility and perceptual selection. Journal of Experimental Social Psychology, 22, 293-311.

Davison, A. C., \& Hinkley, D. V. (1997). Bootstrap methods and their application. Cambridge, UK: Cambridge University Press.

De Houwer, J., Hermans, D., Rothermund, K., \& Wentura, D. (2002). Affective priming of semantic categorization responses. Cognition and Emotion, 16, 643-666.

Frings, C., Rothermund, K., \& Wentura, D. (2007). Distractor repetitions retrieve previous responses to targets. Quarterly Journal of Experimental Psychology, 60, 1367-1377.

Golubickis, M., Falbén, J. K., Cunningham, W. A., \& Macrae, C. N. (2018). Exploring the selfownership effect: Separating stimulus and response biases. Journal of Experimental Psychology: Learning, Memory and Cognition, 44, 295-306.

Henik, A., Friedrich, F. J., \& Kellog, W. A. (1983). The dependence of semantic relatedness effects upon prime processing. Memory \& Cognition, 11, 366-373.

Hommel, B. (2004). Event files: Feature binding in and across perception and action. Trends in Cognitive Sciences, 8, 494-500.

Humphreys, G. W., \& Sui, J. (2016). Attentional control and the self: The self-attention network (SAN). Cognitive Neuroscience, 7, 5-17.

Keyes, H., \& Brady, N. (2010). Self-face recognition is characterized by "bilateral gain" and by faster, more accurate performance which persists when faces are inverted. The Quarterly Journal of Experimental Psychology, 63, 840-847. 
Kiefer, M. (2007). Top-down modulation of "automatic" processes: A gating framework. Advances in Cognitive Psychology, 3, 289-306.

Kiefer, M., Adams, S. C., \& Zovko, M. (2012). Attentional sensitization of unconscious visual processing: Top-down influences on masked primes. Advances in Cognitive Psychology, 8 , $50-61$.

Klauer, K. C., \& Musch, J. (2002). Goal-dependent and goal-independent effects of irrelevant evaluations. Personality and Social Psychology Bulletin, 28, 802-814.

Kuiper, N. A., \& Rogers, T. B. (1979). Encoding of personal information: Self-other differences. Journal of Personality and Social Psychology, 37, 499-514.

Macrae, C. N., Visokomogilski, A., Golubickis, M., Cunningham, W. A., \& Sahraie, A. (2017). Self-relevance prioritizes access to visual awareness. Journal of Experimental Psychology: Human Perception and Performance, 43, 438-443.

Maxfield, L. (1997). Attention and semantic priming: A review of prime task effects. Consciousness \& Cognition, 6, 204-218.

Moors, A., \& De Houwer, J. (2006). Automaticity: A conceptual and theoretical analysis. Psychological Bulletin, 132, 297-326.

Naccache, L., Blandin, E., \& Dehaene, S (2002). Unconscious masked priming depends on temporal attention. Psychological Science, 13(5), 416-424.

Pinheiro, J., Bates, D., DebRoy, S., Sarkar, D., \& R Development Core Team. (2015). nlme: Linear and nonlinear mixed effects models. The Comprehensive R Archive Network (CRAN), Vienna, Austria.

Reuther, J., \& Chakravarthi, R. (2017). Does self-prioritization affect perceptual processes? Visual Cognition, 25, 381-398. 
Shapiro, K. L., Caldwell, J., \& Sorensen, R. E. (1997). Personal names and the attentional blink: A visual “cocktail party” effect. Journal of Experimental Psychology: Human Perception and Performance, 23, 504-514.

Siebold, A., Weaver, M. D., Donk, M., \& van Zoest, W. (2016). Social salience does not transfer to oculomotor visual search. Visual Cognition, 23, 989-1019.

Stein, T., Siebold, A., \& Zoest, M. V. (2016). Testing the idea of privileged awareness of selfrelevant information. Journal of Experimental Psychology: Human Perception and Performance, 42, 1-16.

Sui, J., He, X., \& Humphreys, G. W. (2012). Perceptual effects of social salience: Evidence from self-prioritization effects on perceptual matching. Journal of Experimental Psychology: Human Perception and Performance, 38, 1105-1117.

Sui, J., \& Humphreys, G. W. (2017). The ubiquitous self: What the properties of self-bias tell us about the self. Annals of the New York Academy of Sciences, 1396, 222-235.

Sui, J., Liu, M., \& Mevorach, C., \& Humphreys, G. W. (2013). The salient self: The left intraparietal sulcus responds to social as well as perceptual salience after self-association. Cerebral Cortex, 4, 1060-1068.

Sui, J., Sun, Y., Peng, K., \& Humphreys, G. W. (2014). The automatic and the expected self: Separating self- and familiarity biases effects by manipulating stimulus probability. Attention, Perception, \& Psychophysics, 76, 1176-1184.

Symons, C. S., \& Johnson, B. T. (1997). The self-reference effect in memory: A meta-analysis. Psychological Bulletin, 121, 371-394.

Wade, G. L., \& Vickery, T. J. (in press). Target self-relevance speeds visual search responses but does not improve search efficiency. Visual Cognition. 